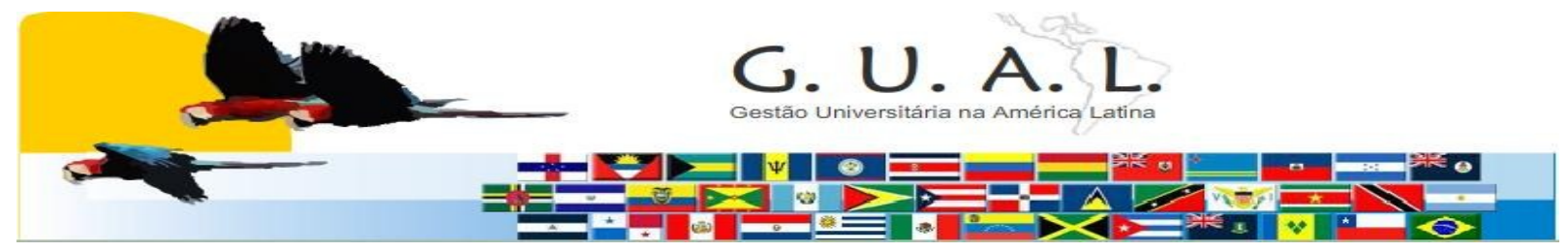

ISSN 1983-4535

\title{
COMPETENCIAS PROFESIONALES DE LOS ACADÉMICOS EN LA EDUCACIÓN SUPERIOR
}

\author{
Guillermo Yáber Oltra, Ph.D. \\ Universidad Simón Bolívar - Venezuela \\ gyaber@usb.ve \\ Carmen Alfonzo, Mestre \\ Universidad Simón Bolívar - Venezuela \\ calfonzo@usb.ve
}

\begin{abstract}
RESÚMEN
El desarrollo profesional de los profesores y los líderes universitarios es un proceso continuo en la educación superior. Docencia, investigación, servicio, desarrollo personal y gestión son las competencias profesionales que un académico puede desarrollar durante su carrera. Se desarrolló un instrumento de auto-reporte que permite analizar la discrepancia entre la maestría que el personal académico y directivo aprecia de sus competencias académicas, personales y de gestión así como la importancia que la institución les asigna. Se presentan dos casos de instituciones donde estos instrumentos se aplicaron a gerentes y académicos. Se analiza el uso de este instrumento para el desarrollo de políticas y prácticas de gestión del talento académico y gerencial de las instituciones de Educación Superior.
\end{abstract}

Palabras claves: Competencias. Académicos. Desarrollo de carrera. 


\section{INTRODUCCIÓN}

El éxito de una organización en el cumplimiento de su misión depende del acoplamiento de las metas y objetivos de las unidades que la componen, con las competencias requeridas para lograr las metas y cumplir con la misión de la institución. (Prahalad y Hamel, 1990)

En las Instituciones de Educación Superior (IES) y particularmente en las universidades, el cumplimiento de su misión, requiere la determinación de las competencias que debe desarrollar el talento humano de sus comunidades (profesores, estudiantes, personal de apoyo, directivos), para alcanzar las metas establecidas en sus planes. Las metas se formulan en los planes estratégicos, operativos y de gestión para luego documentarse en las memorias y cuentas institucionales que incluyen los logros individuales, grupales, de las unidades organizacionales y de la universidad en su conjunto según cada caso (Figura 1.)

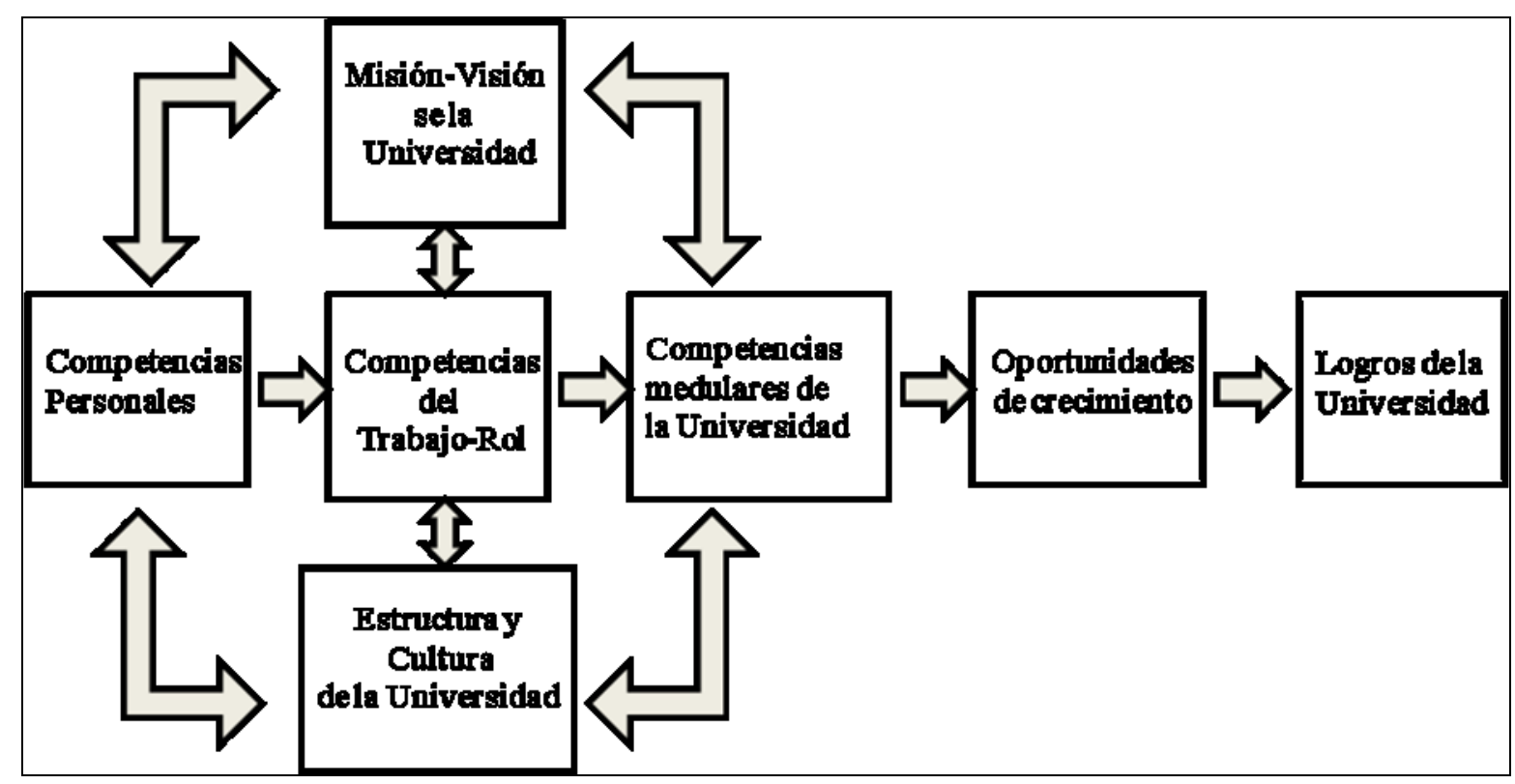

Figura 1: Modelaje de competencias.

Fonte: Adaptado de Punnitamai, (2002)

La generación del conocimiento (Investigación), la formación y acreditación del talento humano (Docencia), y la prestación de servicios (Extensión) constituyen las competencias medulares de una Institución de Educación Superior. El énfasis proporcional que las universidades asignan a cada una de estas competencias contribuye a definir el perfil de la institución. Existen universidades cuyo énfasis mayor es la investigación, en otras la 
extensión o servicio y la mayoría pone el mayor énfasis en la actividad de docencia. Estas combinaciones exigen diferentes énfasis en las competencias que debe desarrollar su personal académico.

Planificación, Organización, Dirección y Control de los recursos para lograr metas (Gestión) y el mejoramiento del bienestar individual (Desarrollo Personal), son competencias que desarrolla el talento humano de la institución para asegurar el éxito de la misión universitaria. En la agenda de la UNESCO para la segunda década del siglo XXI, en el punto concerniente a "Acceso, Equidad y Calidad", el numeral 15 especifica que "las instituciones de educación superior deben invertir en el entrenamiento de la facultad y del personal en los sistemas de enseñanza y aprendizaje que se transforman” (UNESCO, 2009).

En la diversidad de instituciones de educación superior de las Américas, existe una variedad de opciones para el desarrollo de su personal académico y de gestión que oscilan desde la ausencia total de éstos, hasta programas complejos de formación organizados bajo una vice-rectoría dirección, gerencia o departamento. En la medida en que la institución formaliza más la atención al desarrollo del personal académico se hace necesario el uso de recursos para detectar necesidades de entrenamiento y desarrollo. Un ejemplo lo constituyen los instrumentos de valoración de necesidades donde el personal responde a las opciones disponibles o indica en forma abierta su opinión sobre necesidades de entrenamiento y desarrollo.

Las universidades pueden también realizar estudios de las competencias organizacionales requeridas para alcanzar sus metas y objetivos, evaluarlas en su personal y luego desarrollar los programas de entrenamiento y desarrollo que contribuyan a disminuir las brechas entre lo que la Institución considera importante y el desempeño actual del personal académico. Una forma de hacerlo se presenta seguidamente.

\section{COMPETENCIAS ACADÉMICAS Y GESTIÓN UNIVERSITARIA}

En la diversidad de instituciones de Educación Superior del continente americano se encuentra personal de la institución que ha sido contratado originalmente como personal académico y luego es promovido o ha ganado una posición como gestor universitario. Por otra parte podemos encontrar en nuestras instituciones, personal que ha sido contratado para realizar trabajos de naturaleza administrativa, técnica o ambas y puede ascender a diferentes posiciones de gestión administrativa en las universidades.

Rev. GUAL., Florianópolis, v.4, n. 1, p.129-139, jan/abr. 2011 
Una carrera como personal académico de una institución de educación superior demanda que la persona se vuelva progresivamente competente para realizar actividades de docencia, investigación y extensión. Un sub-conjunto de los profesores suelen desarrollan carreras que incluyen la gestión académica, administrativa o híbrida y todos requieren de un desarrollo personal que armonice con su carrera profesional.

Competencias académicas (Anexo 1), es un instrumento de auto-reporte de la apreciación de la importancia y el dominio que el personal académico de una institución de educación superior tiene sobre sus necesidades de entrenamiento en las competencias requeridas en investigación, docencia extensión, gerencia académica y desarrollo personal. La Docencia alude a las actividades académicas que realizan los profesores para la capacitación técnica, formación integral, desarrollo y especialización profesional; así como de formación en investigación para estudiantes de grado y postgrado en instituciones de educación superior.

En investigación se incluye lo concerniente a la generación de conocimientos y su difusión en ambientes académicos, profesionales y medios de comunicación masivos que redunden en el beneficio de la sociedad. La extensión contempla las actividades de servicio interno o externo para prestar asistencia, solucionar problemas o desarrollar aplicaciones, sobre asuntos de interés para la universidad o la sociedad. Con la gerencia académica, la persona ejerce el liderazgo y la gestión para conducir al logro de metas en unidades académicas o instituciones de educación superior. El desarrollo de la maestría personal y gestión de la carrera académica es la dimensión con la que los profesores se hacen progresivamente competentes en su desarrollo personal, aspecto relevante en el desarrollo de su carrera académica. (Figura 2)

Para el modelaje de las competencias (Mirabile, 1997; Markus, Cooper-Thomas y Allpress, 2005), se solicitó a académicos “ejemplares” y con experiencia en entrenamiento, desarrollo y evaluación de personal, para que redactaran frases alusivas a unidades de competencia que correspondieran a cada una de las competencias de la carrera académica en las cuales se habían puesto previamente de acuerdo (docencia, investigación, extensión, gerencia académica y desarrollo personal). Estas frases fueron depuradas en su redacción y contenido por tres expertos que realizaron la redacción final. El instrumento incluye 38 enunciados de unidades de competencia que fueron colocados de acuerdo a los expertos, en cada una de las cinco competencias. La persona que responde al instrumento aprecia la 
importancia que tiene cada unidad de competencia para su institución y sobre el dominio que el propio profesional aprecia sobre esa unidad de competencia. Esta apreciación se realiza con escalas que van del 1 al 5 tanto para la importancia como para el dominio.

Puede ocurrir que alguna unidad de competencia no aplique en términos de importancia para una institución (por ejemplo instituciones dedicadas a la docencia pueden no atribuir importancia a la investigación) es por ello que en la escala de importancia se agregó una columna de no aplica (n/a), para aquéllos casos en que la unidad de competencia no sea pertinente. El instrumento puede analizarse individual o grupalmente de acuerdo a los intereses de quienes lo aplican. Grupalmente proporciona información valiosa sobre necesidades de entrenamiento del personal académico en forma general. En forma individual, es un instrumento útil para la evaluación y desarrollo de los portafolios del personal académico.

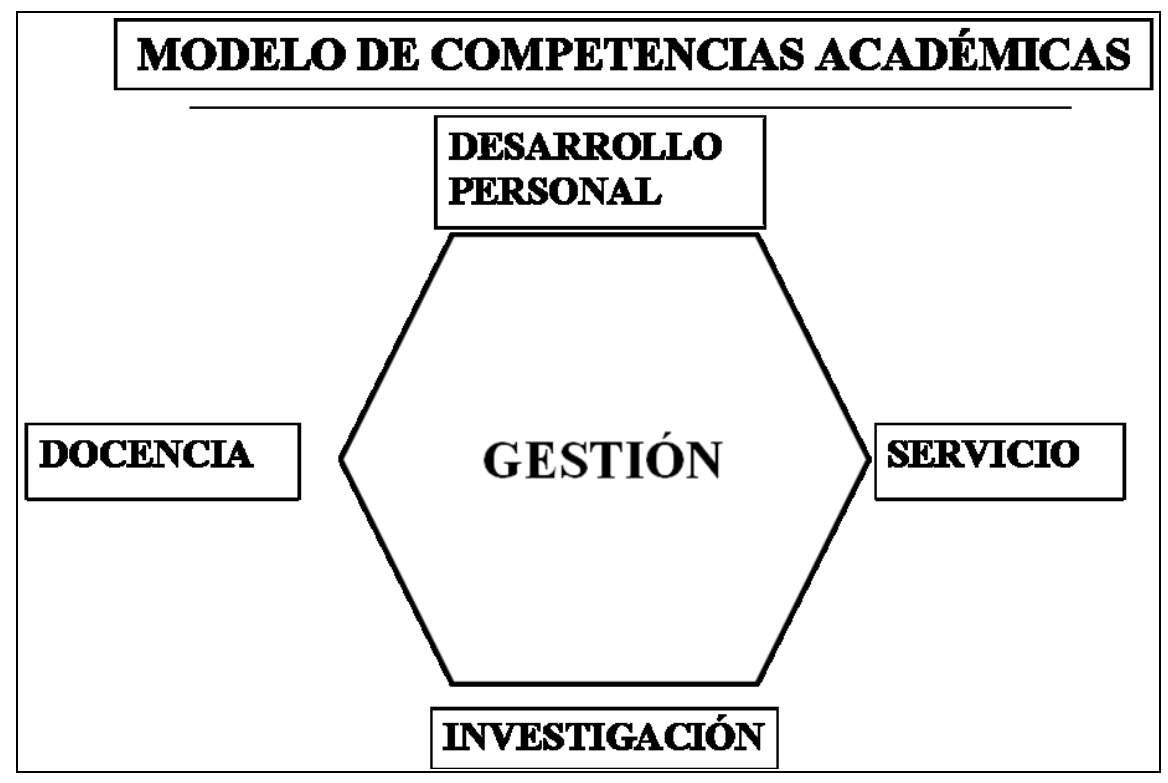

Figura 2 - Competencias Académicas

El instrumento puede adaptarse a la diversidad institucional. Por ejemplo una universidad que no hace investigación puede eliminar los enunciados correspondientes a esa competencia. Por otra parte, dentro de una competencia (por ejemplo docencia) pueden incluirse o eliminarse unidades de competencia. Por ejemplo si en la institución no se realizan exámenes orales, la unidad de competencia correspondiente puede eliminarse. La aplicación de este instrumento en dos casos se presenta a continuación. 


\subsection{Competencias académicas: estudio de casos}

\subsubsection{Instituto de gestión y liderazgo universitario}

La Organización Universitaria Interamericana (OUI), realiza anualmente el programa IGLU, de entrenamiento y desarrollo de gestión y liderazgo universitario (OUI-IOHE, 2009). Este programa se ofrece para directivos universitarios de gerencia superior y media de las Instituciones de Educación Superior de las Américas a través de varias regiones geográficas entre las cuales se encuentra la región Caribe que atiende fundamental pero no exclusivamente a los directivos de las universidades de Venezuela, República Dominicana, Puerto Rico, Cuba, Haití y Guyana.

El programa contempla una primera parte de talleres intensivos de tres semanas de duración donde los participantes de estos países intercambian con pares sus experiencias como gestores universitarios, mientras adquieren y fortalecen conocimientos, habilidades y destrezas en aspectos como: planificación estratégica; liderazgo; conflicto y negociación; gestión de unidades universitarias; historia y estatus actual de la educación universitaria interamericana; y cooperación internacional, entre otros asuntos.

En este contexto formulan un plan personal de cambio que incluye el mejoramiento o desarrollo de una o más de las unidades de competencias vinculadas a la docencia, investigación, extensión, gestión o desarrollo personal. Para ello completan el instrumento de competencias académicas y formulan un sencillo plan de cambio a cumplirse en el siguiente año. De acuerdo a los testimonios de estos directivos, la actividad de responder al instrumento ayuda en el plan de carrera profesional. Todos encuentran oportunidades para desarrollar sus competencias.

Algunos "descubren" necesidades de desarrollo, muchos reflexionan sobre la discrepancia entre la importancia que la institución asigna a las competencias y el grado de desarrollo que los académicos aprecian en sí mismos. Para todos resulta una experiencia significativa en el desarrollo de su carrera en la academia. Algunos académicos reportan que lo han empleado dentro de actividades de "Coaching", "Mentoría" u otras variantes de relación de ayuda con pares de sus propias unidades académicas o de otras dentro de la institución.

En estos casos el asesor ayuda al otro profesor a identificar sus necesidades de entrenamiento y desarrollo y preparar un plan de acción. Algunos participantes han señalado

Rev. GUAL., Florianópolis, v.4, n. 1, p.129-139, jan/abr. 2011 
sin embargo, que el instrumento está orientado a personal académico que cumple funciones de dirección, pero no considera a muchos gerentes y directores universitarios que no cumplen actividades académicas.

Esto ha llevado a formular un segundo instrumento de "competencias de gestión universitaria" que se encuentra en fase de prueba y que incluye las competencias de: gestión general, gestión de talento humano, gestión financiera, gestión de proyectos, gestión de operaciones y rendición de cuentas (Alfonzo, Yáber, y Farrera, 2010). Una ventaja de ambos instrumentos es su carácter genérico, que debe modificarse para adaptarse a las distintas realidades de las instituciones de educación superior de las Américas ya que las competencias son situacionales y dependen de cada institución a pesar que existan muchos aspectos comunes en las universidades.

En otras ocasiones, el instrumento de competencias académicas puede emplearse para explorar la importancia y dominio que el personal directivo de una institución aprecia de sus propias competencias. Presentamos un ejemplo a continuación.

\subsubsection{Competencias en directivos académicos de una universidad de la región Caribe}

Directivos académicos $(n=21)$, responsables de programas y unidades académicas de una universidad privada de la región Caribe, respondieron durante una sesión de taller sobre desarrollo gerencial y liderazgo, el instrumento de competencias académicas. En la figura 3 se puede visualizar el perfil de importancia y dominio de las competencias académicas tal y como lo apreció colectivamente este grupo.

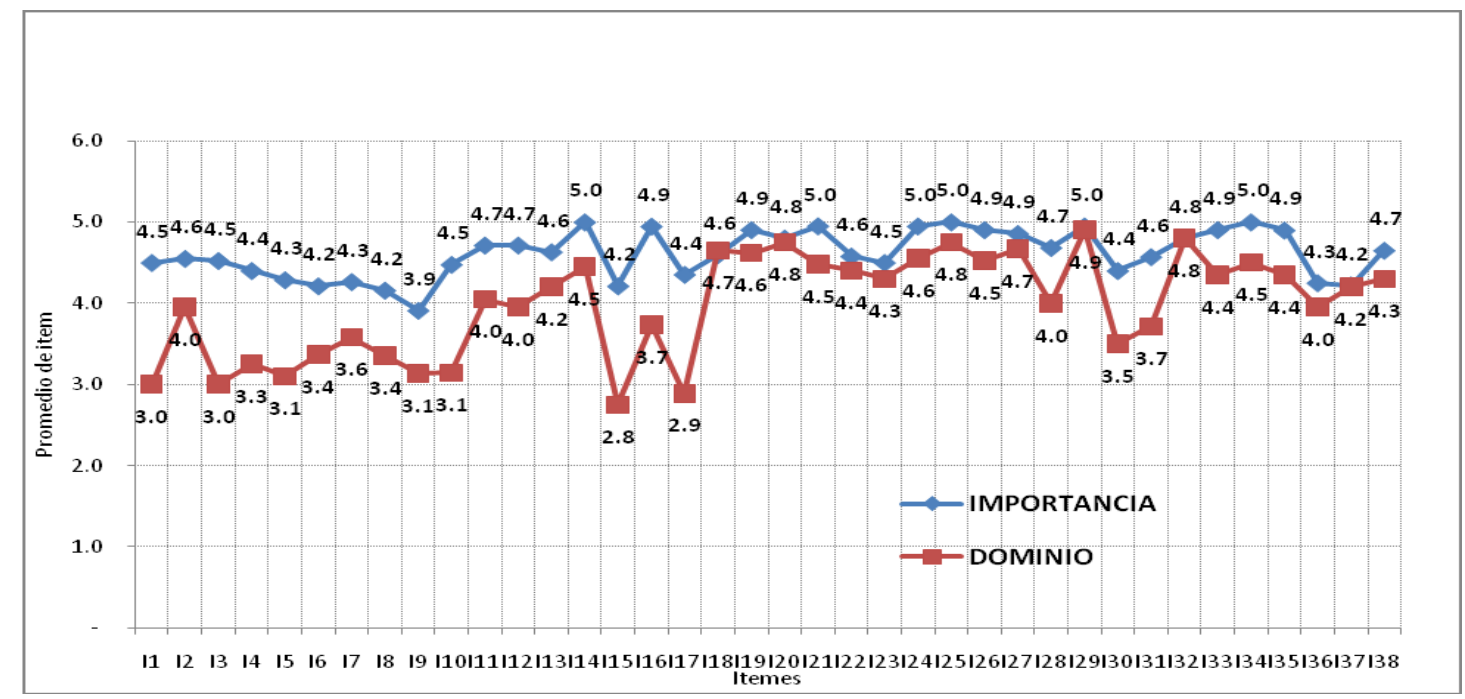

Figura 3. Importancia y dominio de competencias académicas

Rev. GUAL., Florianópolis, v.4, n. 1, p.129-139, jan/abr. 2011 
Al examinar visualmente el gráfico se pueden extraer varias conclusiones. El perfil promedio de dominio se encuentra por debajo del perfil promedio de importancia salvo en cinco unidades de competencia $(18,20,29,32$ y 37), donde la importancia y el dominio tienen promedios iguales. El nivel de importancia promedio oscila desde 3 (unidad 1) hasta 5 en seis unidades de competencia $(14,21,24,25,29$ y 34). De acuerdo a lo reportado por el grupo. Existe consenso en estos directivos sobre la máxima importancia de esas seis unidades para los profesores de esta universidad. Existen unidades donde la discrepancia llega a ser hasta de 1,5 puntos entre importancia y dominio $(1,3,15,17)$. Es posible también realizar análisis estadísticos y obtener el grado de significación y el tamaño de la discrepancia, realizando por ejemplo una prueba " $\mathrm{t}$ " de student para grupos emparejados. Se pueden revisar las diferencias comparando competencias, es decir, determinar por ejemplo si la competencia de extensión está más desarrollada en el personal que la de investigación.

De acuerdo a las prioridades de la institución se pueden realizar distintos análisis y orientar entonces los planes de desarrollo del personal académico y de dirección universitaria.

\section{CONCLUSIONES}

El desarrollo del talento en el personal académico y directivo de una Institución de Educación Superior es un factor clave para la competitividad. El entrenamiento y desarrollo por competencias es una opción que tienen las instituciones para llevar adelante este desarrollo. Alinear las competencias de los académicos con las competencias medulares de la universidad contribuye a su crecimiento, posicionamiento competitivo y logros. Se presentó una técnica de modelaje de competencias que obtuvo como resultado un instrumento de autoreporte donde el personal académico y directivo pondera la importancia y dominio de las competencias medulares y de apoyo para el éxito en la academia. El instrumento es de carácter abierto y puede modificarse de acuerdo a las peculiaridades de cada universidad, donde cada institución puede agregar y eliminar competencias como unidades de competencia. Con los ejemplos se ilustró su aplicación en el portafolio profesoral como herramienta de desarrollo y para evaluar colectivamente las competencias en un grupo de directivos provenientes de una universidad. Se presentaron las descripciones de las competencias de docencia, investigación, extensión, gerencia académica y desarrollo personal. Con estos insumos, las universidades pueden gestionar programas que contribuyan al desarrollo de las competencias del talento humano profesoral y directivo universitario. 


\section{REFERENCIAS}

ALFONZO, C.; YÁBER, G; FARRERA, M. Competencias de gestión universitaria. Manuscrito en preparación, (2010).

MARKUS, L.H.; COOPER-THOMAS, H.D; ALLPRESS, K.N. Confounded by competencies? An evaluation of the evolution and use of competency models. New Zealand Journal of Psychology34 (2), p. 117-126, 2005.

MIRABILE, R. Everything you wanted to know about competency modeling. Training \& Development, p. 73-77, ago, 1997.

OUI-IOHE; Instituto de gestión y liderazgo universitario, 2009. Recuperado desde http://www.oui-iohe.org/iglu/, el 20 de diciembre de 2010.

PRAHALAD, C.K; HAMEL, G. The core competence of the corporation. Harvard Business Review, 68 (3), p. 79-91, 1990.

PUNNITAMAI, W. The application of competency modeling for human resource management. A holistic inquiry, Thai journal of public administration, p. 113-132, set/dez, 2002.

UNESCO. Comunicado de la conferencia mundial de la educación superior, 2009.

Recuperado desde http://www.unesco.org/education/WCHE2009/comunicado_es.pdf el 20 de diciembre de 2010 


\section{ANEXO I - COMPETENCIAS ACADÉMICAS}

\begin{abstract}
Nombre y
apellido:
\end{abstract}

Institución:

\author{
Profesor(a)/investigador(a)
}

en la disciplina de:

\section{COMPETENCIAS ACADÉMICAS}

Indique el nivel de importancia y el grado en que usted domina cada una de las $\mathbf{3 8}$ unidades de competencia académicas que corresponden a Investigación, extensión, desarrollo personal, gerencia académica y docencia. El grado de importancia es la que usted cree que le asigna la institución para la cual trabaja o en la última para la que prestó servicios. ¡Gracias! 1= Nada de importancia o dominio. 5= Total importancia o dominio. n/a= No aplica.

\begin{tabular}{|c|c|c|}
\hline Competencia de: & IMPORTANCIA & DOMINIO \\
\hline (A) INVESTIGACIÓN & $123 \quad 3455 \mathrm{n} / \mathrm{a}$ & 12345 \\
\hline 01.- Formular proyectos de investigación & 000000 & 00000 \\
\hline 02.- Desarrollar productos o servicios para el mejoramiento o innovación & 00000 & 00000 \\
\hline 03.- Desarrollar líneas de investigación & 00000 & 00000 \\
\hline 04.- Difundir resultados de investigación en ambientes académicos & 00000 & 00000 \\
\hline 05.- Difundir resultados de investigación en medios de comunicación & 00000 & 00000 \\
\hline 06.- Analizar datos con enfoque cuantitativo & 00000 & 00000 \\
\hline 07.- Escribir para publicar en revistas especializadas & 00000 & 00000 \\
\hline 08.- Analizar datos con enfoque cualitativo & 00000 & 00000 \\
\hline 09.- Desarrollar trabajos de ascenso & 00000 & 00000 \\
\hline 10.- Arbitrar trabajos académicos (proyectos, artículos, libros, ponencias) & 000000 & 00000 \\
\hline (B) EXTENSIÓN & $\begin{array}{llllll} & 2 & 3 & 4 & 5 & \mathrm{n} / \mathrm{a}\end{array}$ & 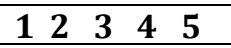 \\
\hline 11.- Formular proyectos de aplicación o servicio & 000000 & 00000 \\
\hline 12.- Formular proyectos de trabajo voluntario & $0 \bigcirc \bigcirc \bigcirc 0$ & 00000 \\
\hline 13.- Actuar como vocero de la universidad ante los medios de comunicación & 00000 & 00000 \\
\hline 14.- Formular proyectos de educación continua & $0 \bigcirc 0 \bigcirc 0$ & 00000 \\
\hline 15.- Gestionar la recaudación de fondos para la institución (Fund-Raising) & 00000 & 00000 \\
\hline 16.- Gestionar proyectos de cooperación académica inter-institucional & 00000 & 00000 \\
\hline 17.- Cabildear frente a organismos públicos a nombre de la universidad & 000000 & 00000 \\
\hline (C) DESARROLLO PERSONAL & $\begin{array}{llllll}1 & 2 & 3 & 4 & 5 & \mathrm{n} / \mathrm{a}\end{array}$ & $\begin{array}{lllll}1 & 2 & 3 & 4 & 5 \\
\end{array}$ \\
\hline 18.- Comunicarse inter-personalmente en forma efectiva & 000000 & 00000 \\
\hline 19.- Comunicarse formalmente en ambientes académicos & 00000 & 00000 \\
\hline 20.- Comportarse asertivamente & 00000 & 00000 \\
\hline 21.- Planificar la carrera académica & 00000 & 00000 \\
\hline 22.- Gestionar la vida personal eficazmente & 00000 & 00000 \\
\hline 23.- Gestionar conflictos y negociar & 000000 & 00000 \\
\hline (D) GERENCIA ACADÉMICA & $\begin{array}{llllll}1 & 2 & 3 & 4 & 5 & \mathrm{n} / \mathrm{a}\end{array}$ & 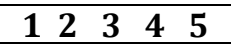 \\
\hline 24.- Gestionar unidades académicas & 000000 & 00000 \\
\hline 25.- Supervisar profesores, preparadores o personal de apoyo & $0 \bigcirc 0 \bigcirc 0$ & 00000 \\
\hline 26.- Planificar estratégica, táctica y operativamente & 00000 & 00000 \\
\hline 27.- Manejar grupos y equipos de trabajo & 00000 & 00000 \\
\hline 28.- Gestionar cambios en unidades organizacionales & 000000 & 00000 \\
\hline (E) DOCENCIA & $\begin{array}{llllll} & 2 & 3 & 4 & 5 & \mathrm{n} / \mathrm{a}\end{array}$ & \begin{tabular}{lllll|}
1 & 2 & 3 & 4 & 5 \\
\end{tabular} \\
\hline 29.- Diseñar asignaturas presenciales & 000000 & 00000 \\
\hline 30.- Diseñar asignaturas virtuales & 00000 & 00000 \\
\hline 31.- Diseñar asignaturas híbridas (semi-presenciales) & 00000 & 00000 \\
\hline 32.- Manejar sesiones de clase & 00000 & 00000 \\
\hline 33.- Evaluar el desempeño estudiantil & 00000 & 00000 \\
\hline 34.- Aplicar estrategias de enseñanza/aprendizaje & 00000 & 00000 \\
\hline 35.- Diseñar evaluaciones escritas & 00000 & 00000 \\
\hline 36.- Diseñar evaluaciones orales & 00000 & 00000 \\
\hline 37.- Redactar reportes de asignaturas/cátedra & 00000 & 00000 \\
\hline 38.- Tutoría de estudiantes en tesis, pasantías o aprendizaje en el servicio & 000000 & 00000 \\
\hline
\end{tabular}




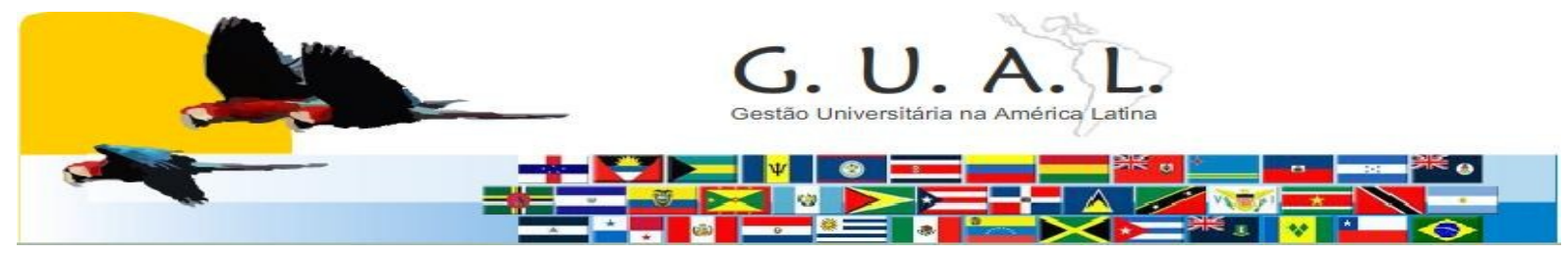

ISSN 1983-4535

\title{
SCHOLARS' PROFESSIONAL COMPETENCIES IN HIGHER EDUCATION
}

\author{
Guillermo Yáber Oltra, Ph.D. \\ Carmen Alfonzo, Master \\ Organización Universitaria Interamericana - OUI \\ Universidad Simón Bolívar - Venezuela \\ centroiglu-caribe@usb.ve
}

\begin{abstract}
The professional development of faculty and higher education leaders is a continuous process in higher education. Teaching, research, service, personal development and management are the professional competencies that a scholar may develop during his/her career. A self-report instrument was developed to allow a discrepancy analysis between the perceptions scholars and managers have of the mastery of their academic, personal and management competencies, and the relative importance that the institution assigns to those same competencies. Two institutional cases are presented, in which these instruments are administered to both scholars and managers. The use of this instrument is analyzed for the development of policies and practices of academic and managerial talent within higher education institutions.
\end{abstract}

Keywords: Competencies. Faculty. Career development. 